\title{
Las brechas entre la política educativa y las prácticas de los actores escolares en el contexto de la pandemia. El caso de los bachilleratos rurales en México
}

\author{
Carlota Guzmán Gómez \\ Universidad Nacional Autónoma de México (UNAM)
}

Resumen. En este artículo se analizan las diferencias entre los lineamientos de la política educativa desplegados durante la pandemia de la COVID-19, a través del programa Aprende en casa, y las prácticas de los actores escolares. El estudio se enfoca en el nivel medio superior en contextos rurales de alta vulnerabilidad en México, específicamente la modalidad de telebachilleratos comunitarios. Desde una perspectiva metodológica que integra una fase documental y otra cualitativa, se busca visibilizar el destino de la política educativa, tomando en cuenta la perspectiva de los actores y las especificidades del contexto. Se encontró que, a pesar del discurso triunfalista del secretario de Educación, las/os responsables de los planteles y las/os docentes consideran que fue inadecuado para las condiciones y necesidades del medio rural. Por este motivo, enfrentaron con sus propios recursos y estrategias la educación a distancia de emergencia.

Palabras clave: política educativa; Estado y educación; educación rural

As lacunas entre a política educacional e as práticas dos atores escolares no contexto da pandemia. O caso das escolas de ensino médio rurais no México

Resumo. Este artigo analisa as diferenças entre as diretrizes de política educacional implantadas durante a pandemia da COVID-19, através do programa Aprende en Casa, e as práticas dos atores escolares. O estudo enfoca a educação de nível médio superior em contextos rurais altamente vulneráveis no México, especificamente a modalidade de telecursos, chamados "telebachilleratos comunitarios". Sob uma perspectiva metodológica que integra uma fase documental e outra qualitativa, procura dar visibilidade ao destino da política educacional, considerando a perspectiva dos atores e as especificidades do contexto. Verificou-se que, apesar do discurso triunfalista do secretário de Educação, as/os responsáveis pelas escolas e as/os docentes consideram que era inadequado para as condições e necessidades do meio rural. Por esta razão, enfrentaram a educação à distância de emergência com seus próprios recursos e estratégias.

Palavras-chave: política educacional; Estado e educação; educação rural.

The gap between education policy and the practices of actors within school systems in the context of the pandemic. The case of rural high schools in Mexico

Abstract. This article analyzes the differences between education policy guidelines deployed during the COVID-19 pandemic, through the Aprende en casa (Learn at Home) program and the practices of actors within school systems. This study focuses on upper secondary education in highly vulnerable rural contexts in Mexico, specifically on community distance-learning high schools. From a methodological perspective that consists of a documental and a qualitative phase, the aim is to shed light on the direction of education policy, taking into account the perspective of the actors involved as well as the specificities of the context. The study found that, despite the triumphalist discourse used by the Secretary of Education, both those responsible for the schools and the teachers do not consider that it was adequate for the needs and conditions of rural areas. For this reason, they had to face the emergency of remote education with their own means and strategies.

Keywords: education policy; State and education; rural education. 


\section{Introducción}

Al poco tiempo de que iniciara la pandemia por COVID-19 y el subsecuente confinamiento, fue evidente que los sistemas educativos recibirían un fuerte golpe. Es sabido que la mayoría de los países optó por suspender las clases presenciales para sustituirlas por la educación a distancia. Para las poblaciones más pobres que no cuentan con dispositivos tecnológicos y acceso a internet, este hecho obstaculizó la continuación de los procesos de enseñanza y de aprendizaje (ONU, 2020). Está ampliamente documentado que la pandemia no solo visibilizó las desigualdades sociales y educativas preexistentes, sino que las exacerbó (CEPAL-UNESCO, 2020; ONU, 2020).

Ante dicho contexto, México estableció la educación a distancia de emergencia mediante el programa Aprende en casa, el cual se basa en la transmisión de contenidos curriculares por radio y televisión, apoyados en internet. El programa se dirige a la población que cursa desde preescolar hasta el nivel medio superior. De esta manera, la educación a distancia tuvo que instalarse en un país que tiene un acceso muy limitado a las tecnologías de la información y la comunicación (TIC). A nivel mundial, México ocupa el lugar 69 respecto al acceso a TIC (World Economic Forum [WEF], 2016); en América Latina, se coloca por debajo de Chile, Barbados, Uruguay, Costa Rica, Panamá y Colombia. Esta situación se explica si consideramos que en México solo $44.3 \%$ de los hogares cuenta con computadora y $56.4 \%$ tiene conexión a internet (Instituto Nacional de Estadística y Geografía [INEGI], 2019).

Las dificultades de la educación a distancia de emergencia enfrentadas por docentes, estudiantes y familias en México han salido a la luz a través de columnas de opinión, reportajes y entrevistas. El sector académico también ha documentado y analizado los efectos de la pandemia en la educación y las limitaciones de la modalidad a distancia. Diversas instituciones han realizado importantes eventos, convocado a números temáticos de revistas, publicado artículos (incluso libros); asimismo, han aplicado algunas encuestas para conocer los puntos de vista de la población. A partir de dicha producción, se observan tres núcleos temáticos: 1) consecuencias sociales y educativas de la pandemia; 2) vivencias de estudiantes y docentes en la educación a distancia, y 3) uso y manejo de las nuevas tecnologías. ${ }^{1}$

Los temas derivados de la pandemia son inagotables y seguramente en el futuro inmediato serán una línea de investigación fructífera. La desigualdad educativa es una vertiente que se posiciona central y transversalmente en otras temáticas. Ante este panorama, resulta necesario avanzar con investigaciones puntuales acerca de las afectaciones de la pandemia en cada nivel educativo y en contextos específicos. Para ello, se requiere escuchar las voces de los distintos actores escolares, no solo a nivel anecdótico, sino con estudios profundos y sistemáticos. El campo analítico de la política educativa resulta fértil para no caer en posturas cerradas que aprueben o rechacen las medidas implementadas sin valorar su incidencia a nivel global y en contextos concretos.

\footnotetext{
${ }^{1}$ En un sondeo inicial se identificaron 30 trabajos (10 incluyen todos los niveles, 9 al nivel superior, 3 al medio superior y 8 al básico). Se identificaron 26 encuestas relacionadas con confinamiento sanitario y educación; 12 incluyen el nivel medio superior (7 a estudiantes).
} 
Como parte de una línea de investigación emergente, en este artículo se analizan las diferencias entre los lineamientos de la política educativa desplegados durante la pandemia por COVID-19 y las prácticas de los actores escolares. El estudio se enfoca en la educación media superior (EMS) en contextos rurales de alta vulnerabilidad en México, específicamente la modalidad de telebachilleratos comunitarios (TBC). Se busca visibilizar el destino de la política educativa, considerando la perspectiva de los actores y las especificidades del contexto.

En el estudio se retoma la noción de educación a distancia de emergencia, utilizada para referirse a una estrategia que busca dar continuidad pedagógica y dista de las capacidades y recursos que habría exigido una educación a distancia óptima (Pedró, 2020). Asimismo, se recupera la noción de política de Instituto Nacional para la Evaluación de la Educación, Instituto Internacional de Planeamiento de la Educación y Unesco (INEE, IIEP y Unesco, 2018) que la concibe en dos sentidos:

En primer lugar, como el flujo articulado, regulado y direccionado de bienes, servicios y transferencias que se moviliza desde el Estado para garantizar el derecho a la educación de los niños, niñas y adolescentes desde el nivel preescolar hasta su graduación de la Educación Media Superior [...]. En segundo lugar, la política educativa alude también a la capacidad de los gobiernos para sostener, elaborar estrategias y direccionar la actividad del Estado hacia el logro de los objetivos propuestos (pp. 11-12).

Además, interesa destacar el papel de los actores sociales y políticos, que, desde la concepción de política educativa de Flores-Crespo (2011), recrean de manera constante el curso de las acciones implícitas y explícitas del Gobierno. Esta perspectiva, de acuerdo con dicho autor, lleva a enfocar la política como proceso (policy) y no solo como macroambiente (politics). Se retoman las recomendaciones de Zorrilla y Villa Lever (2003, p.3, citadas por Flores-Crespo, 2011) de atender la EMS, así como abordar las escalas meso y micro de la política educativa para no circunscribirse únicamente al aspecto macrosocial, como ha sido la tendencia de investigación reciente en México.

El aporte de este estudio es el análisis de las escalas macro y meso como referentes para el análisis microsocial de la implementación de la política educativa. Además, se acota a contextos de alta vulnerabilidad económica y se recupera la perspectiva de los actores. Otra contribución consiste en el carácter dinámico del análisis, pues aborda cambios ocurridos en el transcurso de la pandemia. Asimismo, el estudio se ubica en la EMS, escasamente estudiada desde la política educativa, a pesar de que cubre un tramo importante de la escolarización de las/os jóvenes.

\section{Perspectiva metodológica}

Con el fin de contrastar la política educativa con las prácticas de los actores escolares, se distinguen tres escalas: 1) macrosocial: la política educativa federal, plasmada en el programa Aprende en casa; 2) mesosocial: la política estatal hacia la EMS y los TBC, y 3) microsocial: las prácticas escolares de los actores. A partir de dichas escalas se pueden observar expresiones diferenciadas del fenómeno y su vinculación. Acorde con dicha perspectiva, el estudio parte de una fase de investigación documental en la cual se describen los lineamientos de la política educativa frente a la pandemia, y posteriormente se despliega una metodología cualitativa para los niveles meso y microsocial. 
La fase documental se basa en el análisis del programa Aprende en casa en dos etapas, que fueron difundidas en los boletines de la Secretaría de Educación Pública (SEP).

La fase de investigación cualitativa se fundamenta en el interés por recuperar la perspectiva de los actores e incursionar en la dimensión subjetiva, esto es, en sus valoraciones, posicionamientos y puntos de vista (Filck, 2004). Se pone especial énfasis en los contextos sociales y culturales en los que se insertan los actores, desde donde construyen sus significados y despliegan sus prácticas (Vasilachis, 2006; Gibbs, 2012). La atención se focaliza en las/os docentes y responsables de plantel, quienes refieren su propia situación, sus vivencias y sus prácticas, pero también la situación de la comunidad, las/os estudiantes y sus familias. Para abordar la dimensión subjetiva se utilizaron entrevistas semiestructuradas en profundidad, que fueron aplicadas con apoyo de una guía que contempló los siguientes bloques temáticos: 1) condiciones personales y familiares del/la docente; 2) situación de la comunidad, la familia y las/ os estudiantes; 3) formas de trabajo y de comunicación; 4) experiencias y vivencias del trabajo a distancia, y 5) preocupaciones, aprendizajes y perspectivas futuras.

Esquema 1

\section{CATEGORIAS ANALITICAS Y ESTRATEGIAS DE INVESTIGACIÓN}

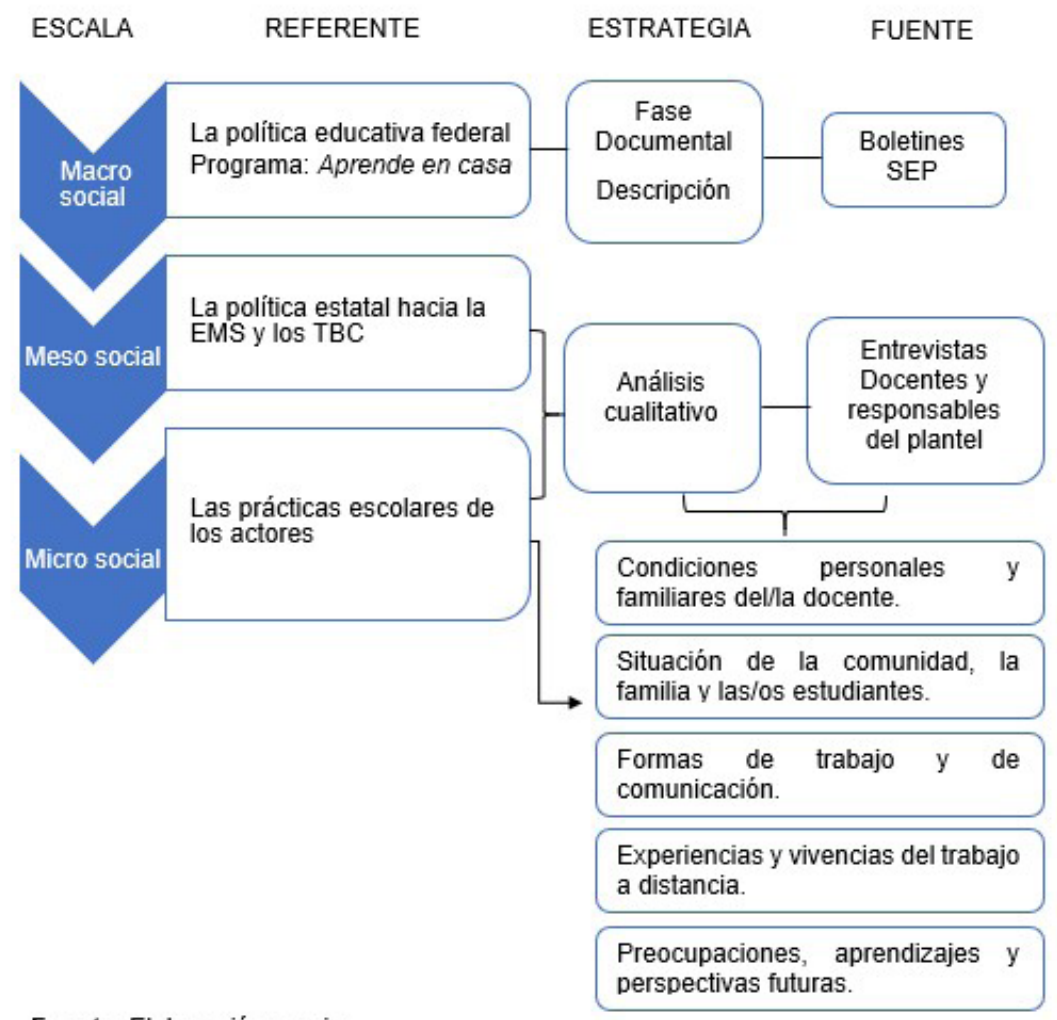

Fuente: Elaboración propia 
La primera etapa del trabajo de campo se realizó durante mayo y junio de 2020. Debido al confinamiento derivado de la pandemia, las entrevistas se realizaron por medio de la plataforma digital Zoom y duraron en promedio dos horas. Con el consentimiento de las/os docentes y el compromiso de mantener el anonimato, quedaron grabadas en esa plataforma. A pesar de las grandes cargas de trabajo de las/os docentes, mostraron amplia disposición para compartir sus experiencias; para ellos, este espacio significó la posibilidad de ser escuchados. Hubiera sido deseable entrevistar también a las/os estudiantes y a sus familias, para que ellas/os hablaran directamente de sus condiciones y sus experiencias, sin embargo, los problemas de conectividad no lo permitieron.

La selección de informantes se realizó a partir de un muestreo teórico, en el cual se establecieron criterios relevantes para dar respuesta a las preguntas de investigación (Vasilachis, 2006; Flick, 2004). Se trata de un estudio de casos múltiples e instrumentales por medio de los cuales, se intenta dar cuenta de una problemática global que no solo compete a los planteles de estudio (Stake, 2010). No se pretende generalizar, sino documentar casos significativos elegidos a partir de criterios contextuales. Para la conformación del universo de estudio, primero se seleccionaron las entidades federativas, posteriormente los planteles y finalmente las/os docentes.

Se eligieron dos entidades federativas: Estado de México y Morelos, las cuales se ubican en el centro del país y comparten condiciones similares de vulnerabilidad económica en los espacios rurales donde se ubican los planteles. En términos del análisis de la política educativa a nivel meso, se buscaron situaciones contrastantes: el Estado de México se caracterizó por poner en marcha dispositivos de apoyo para las/os docentes y establecer lineamientos particulares de gestión desde la Subsecretaría de Educación Media Superior (SEMS); mientras que en Morelos no hubo ningún apoyo. En cada una de estas entidades federativas se eligieron tres TBC ubicados en distintas regiones. En cada TBC se entrevistó a dos docentes, de los cuales una/o desempeña labores directivas y funge también como responsable de plantel. De esta manera, el universo de estudio quedó conformado por 12 docentes (8 mujeres y 4 hombres).

Dado el alargamiento de la pandemia y el inicio de un nuevo ciclo escolar con el programa Aprende en casa II, se realizó una segunda etapa de entrevistas entre noviembre y diciembre de 2020. Estas se aplicaron a responsables de tres planteles del Estado de México y tres de Morelos (5 mujeres y 1 hombre), quienes habían sido entrevistados durante la primera etapa. La indagación se centró en identificar los cambios presentados en las condiciones de los actores y en las prácticas escolares a lo largo de los seis meses. Dicha estrategia se fundamenta en que la pandemia no es un evento que ocurre en un determinado momento, sino que se desarrolla a lo largo del tiempo, y durante ese periodo se generan cambios y ajustes, tanto en la política educativa como en la situación y en las prácticas de los actores.

Las entrevistas fueron transcritas parcialmente y sistematizadas en guías precategorizadas (Huberman y Milles, 2000). En el primer nivel de análisis se buscó, a partir de una matriz temática, identificar tendencias en cada TBC; posteriormente, en cada entidad para poder compararlas entre sí. El segundo nivel correspondió al contraste entre los lineamientos y metas del programa Aprende en casa y lo reportado por las/os docentes. 


\section{La educación media superior en México}

La EMS en México tiene carácter obligatorio, se cursa en tres años y típicamente atiende a la población de entre 15 y 17 años. Dicho nivel está integrado por 5.2 millones de alumnos, 299 mil docentes y 18 mil planteles (INEE, 2019). La tasa de escolarización en este nivel para el mencionado grupo de edad es de 75.2, por debajo de países como Chile (95.5), Bolivia (86.8), Argentina (88.5), Brasil (85.2), Panamá (82.8) y Uruguay (82.1). Además de los problemas de cobertura, en este nivel se presentó una tasa de abandono escolar de 15.2 en el ciclo 2016-2017 (INEE, 2019).

Uno de los problemas más graves de la EMS es la baja cobertura en las zonas rurales; muestra de ello es que $60.9 \%$ del grupo de edad de 15 a 17 años que habita en localidades menores a 2500 habitantes estudia EMS. Mientras tanto, en las que tienen 2500 o más habitantes $79.1 \%$ de ese mismo grupo de edad estudia ese nivel (Comisión Nacional para la Mejora Continua de la Educación [Mejoredu], 2020a).

La SEMS tiene una estructura muy compleja, ya que se organiza a partir de diversas modalidades, con énfasis en lo propedéutico o lo técnico; de régimen público o privado; centralizadas, descentralizadas y autónomas; federales y estatales; así como presenciales y a distancia. En este nivel se busca preparar a los egresados para continuar con los estudios universitarios en diversas disciplinas científicas, tecnológicas y humanísticas, o bien incorporarse al mercado de trabajo. En esta compleja organización de la SEMS se encuentran ubicados los TBC, cuya particularidad es que atienden a una de las poblaciones más vulnerables.

\section{Los telebachilleratos comunitarios}

En el contexto de las grandes desigualdades de la EMS, los TBC fueron creados en 2013 con la finalidad de ampliar la cobertura de este nivel y cumplir con las metas de expansión que se había propuesto el Gobierno para el sexenio 2012-2018. Se pretendía también promover la inclusión y la equidad educativa. Esta modalidad se dirige a las localidades menores a 2500 habitantes donde no exista otro plantel de este mismo nivel a cinco kilómetros a la redonda, ya que se considera que son las zonas con niveles más bajos de cobertura educativa y más altos de marginación económica. En el ciclo escolar 2019-2020 se atendió a un total de 144399 estudiantes en 3306 planteles distribuidos en 31 entidades federativas del país (SEP, SEMS y Dirección General de Bachillerato, 2020).

EI TBC es un modelo propedéutico, escolarizado y presencial que ocupa los espacios de las telesecundarias por las tardes, por lo que no requirió inversión en infraestructura. ${ }^{2}$ Los TBC tienen un mínimo de doce estudiantes y están compuestos por una planta de tres docentes. Cada uno atiende un área curricular y uno de ellos funge simultáneamente como responsable del plantel. Con el fin de promover la inclusión, todos las/os estudiantes reciben una beca, no se cobran colegiaturas y se distribuyen libros de texto gratuitos. ${ }^{3}$

\footnotetext{
${ }^{2}$ La denominación telebachillerato responde a que utilizan los planteles de las telesecundarias.

${ }^{3}$ Las becas recibidas forman parte del programa federal Benito Juárez, dirigido a todos los estudiantes de EMS, por medio del cual reciben 800 pesos mensuales (equivalente a 40 dólares). (Gobierno Federal, 2021) https://www. gob.mx/becasbenitojuarez
} 
Si bien esta modalidad educativa ha permitido estudiar a muchos jóvenes que no habrían podido cursar el bachillerato fuera de su localidad, presenta problemas que ponen en duda el logro de la equidad. Estos se derivan en gran medida de la falta de presupuesto para los TBC, por lo cual operan en condiciones muy precarias. En primer lugar, destaca la falta de plantel propio, le sigue la falta de servicios adecuados, como luz, agua, acceso a internet, ausencia de equipo de cómputo e insumos. No se ofrecen actividades artísticas ni deportivas y las/os docentes tienen condiciones laborales muy precarias: bajos salarios y falta de prestaciones, como seguro médico (Estrada y Alejo, 2019; Guzmán, 2018; Weiss, 2017). Bajo este esquema, las/os jóvenes más vulnerables en términos académicos y económicos se encuentran en desventaja frente a las/os estudiantes de otras modalidades educativas.

\section{El programa Aprende en casa}

Este programa se planteó como una estrategia de la SEP para apoyar las medidas acordadas por la Secretaría de Salud (SSA) y evitar contagios por COVID-19 (SEP, 2020a). Se basó en la transmisión de los contenidos de los libros de texto gratuitos por medio de televisión, radio y con el apoyo de internet, y se utilizaron los canales televisivos públicos, así como la plataforma de la SEMS para albergar los programas y los libros de texto.

El cierre de las escuelas en México se declaró el 23 de marzo de 2020. En un inicio se planteó como un adelanto de las vacaciones, lo cual generó confusión entre la comunidad escolar y las familias. Posteriormente, de una manera más clara, la SEP definió el 20 de abril para retomar las actividades con el programa Aprende en casa. Se intentaba generar certidumbre acerca de la validez de la educación a distancia y asegurar a la población que no se perdería el año escolar. El titular de la SEP aseveraba que se cumpliría tanto con el calendario escolar como con los "aprendizajes esperados", ya que se había alcanzado 75 \% de avance del ciclo escolar 2019-2020 (SEP, 2020b). Ante el alargamiento de la pandemia, el secretario de Educación manifestó que las fechas tenían solo un carácter referencial y que quedaban sujetas a lo que estableciera la SSA. Finalmente, el ciclo escolar tuvo que concluirse a distancia y no pudo llevarse a cabo el periodo de reforzamiento presencial que se tenía previsto al final del ciclo. Reconociendo las dificultades de muchas familias y de las/os estudiantes para llevar a cabo la educación desde casa, se solicitó a las/os docentes no reprobar y tomar en cuenta la comunicación establecida con las/os estudiantes durante esta etapa.

El siguiente ciclo escolar inició el 24 de agosto de 2021 con el programa Aprende en casa II, planteado como continuación de la estrategia. El cambio más importante fue que el Gobierno apostó por la ampliación de la cobertura mediante un convenio con las televisoras privadas, con el argumento de que $94 \%$ de estudiantes tiene acceso a la señal de televisión (Senado de la República, 2020). A través de una amplia difusión mediática, el presidente de la República, Andrés Manuel López Obrador, hizo el anuncio oficial de lo que llamaría un "ejemplo histórico" de la televisión privada del país (Televisa, TV Azteca, Imagen televisión y Grupo Multimedios) cooperando con el Gobierno (Infobae, 2020). 
En la segunda etapa la cobertura alcanzó cuarenta canales nacionales y locales con horarios más amplios. Se sumaron también los canales públicos federales y estatales que venían funcionando desde el inicio, además de diversas televisoras universitarias. Se contó con la radio pública y comunitaria para llegar a las zonas más marginadas, principalmente indígenas, y transmitir la programación en 22 lenguas. Además, se editaron 700 mil cuadernillos para los lugares que no tienen acceso a la radio ni a la televisión (Senado de la República, 2020). El programa contempló la elaboración de 4500 programas de televisión, cuyos contenidos se regirían por los libros de texto elaborados por la SEP sin la intervención de las televisoras. El propio presidente de la República informó que el nuevo programa tendría un costo de 450 millones de pesos (15 pesos por alumno), lo que corresponde únicamente a la operación, ya que no habría ganancias para las empresas (SEP, 2020d). De manera adicional y por medio de un convenio con Google, se logró la gratuidad de sus aplicaciones y se ofrecieron cursos de capacitación a las/os docentes para el manejo del software (SEP, 2020i). ${ }^{4}$

Además de la ampliación de la cobertura televisiva, hubo algunos cambios en la estrategia de comunicación, en los contenidos curriculares y en las recomendaciones para las/os docentes. Para dar a conocer las formas de operación del programa Aprende en casa II, se transmitieron 21 conferencias de prensa presididas diariamente por el secretario de la SEP. Si bien siguieron vigentes los "aprendizajes esperados", se ampliaron los contenidos a temas como "vida saludable" y ética. Al mismo tiempo, se incluyó una programación especial para TBC y el programa en línea Jóvenes en casa, que incluía cursos, webinars y actividades recreativas como lectura, concursos y cine. Se recomendó a las/os docentes tomar en cuenta aspectos emocionales, no exceder las cargas de trabajo y evaluar conforme a la comunicación establecida con las/os estudiantes. Se aclaró que el regreso a clases se haría de forma gradual y escalonada, hasta que cada entidad federativa se considerara en "semáforo epidemiológico en verde", de acuerdo con los parámetros de la SSA.

Al término de la primera etapa del programa Aprende en casa y del desarrollo de su segunda fase, el titular de la SEP manejó un discurso triunfalista que afirmaba que "el aprendizaje a distancia constituye un esquema robusto, equitativo y de calidad" (SEP, 2020c). Sostenía que "con este programa de educación a distancia se está alcanzando a la mayoría de niños, niñas y adolescentes, independientemente de su situación socioeconómica" (SEP, 2020g). También hablaba de un "salto gigante" en materia digital del país, tomando como indicador la creación de 19.5 millones de nuevas cuentas de correo electrónico para estudiantes y 1.2 millones para docentes. Calificaba como un éxito la alianza entre televisoras por llegar a treinta millones de usuarios (SEP, 2020f). Bajo el mismo tenor, mencionó que la educación a distancia permitió a niños, niñas y adolescentes desarrollar nuevas habilidades didácticas, y que el examen para el ingreso a la EMS no registró pérdida de conocimientos (SEP, 2020e). Finalmente, el secretario de Educación también se refería a la revaloración de las/os docentes por parte de las familias y su corresponsabilidad durante esta etapa (Senado de la República, 2020).

\footnotetext{
${ }^{4}$ El costo equivale aproximadamente a 225 mil dólares y por alumno a 0.75 dólares.
} 


\section{La política estatal hacia los telebachilleratos comunitarios durante la pandemia}

En materia educativa, las 32 entidades federativas que componen el país se rigen por los principios generales que establece la SEP como dependencia normativa; sin embargo, quienes operan los sistemas educativos, con algunos márgenes de acción propios, son los gobiernos estatales, a través de sus áreas competentes (SEP, 2020h). En este modelo, el gobierno federal estableció los ajustes al calendario de fin de ciclo, periodo vacacional y reinicio del ciclo escolar. Las/os gobernadoras/es y/o secretarias/os de Educación de cada entidad federativa asumieron la responsabilidad de operar los servicios de educación a distancia y establecer las fechas de regreso a las clases presenciales según los parámetros establecidos por la SSA.

De acuerdo con lo expresado por las/os docentes, desde el cierre de las escuelas por el confinamiento sanitario, en el Estado de México se estableció la plataforma Google Meet como herramienta para la educación a distancia de emergencia. Se propuso para todo el SEMS, independientemente de las condiciones o necesidades de cada modalidad o contexto. La organización por zonas escolares de los TBC operó como base para generar los apoyos necesarios a las escuelas y a las/os responsables de los planteles.

En el caso de Morelos, las/os docentes mencionaron que, si bien se cerraron las escuelas y se ofreció la educación a distancia, fue para acatar la política federal, pero no se establecieron lineamientos particulares para la EMS y mucho menos para los TBC. Cada responsable de plantel definió su propia estrategia y gestionó los apoyos o recursos que consideró necesarios.

\section{7. ¿Qué dicen las/os docentes de telebachilleratos comunitarios?}

\subsection{Los apoyos a nivel estatal}

Las/os docentes de ambas entidades federativas vivieron con angustia el cierre de las escuelas, ya que no se sentían preparadas/os para trabajar a distancia. Les desconcertaba especialmente la falta de claridad en las fechas de inicio, de término del ciclo escolar y del periodo vacacional. La incertidumbre no les permitía planear adecuadamente sus actividades; sin embargo, entendían que se debía al curso que tomaba la pandemia y que había que acatar las medidas sanitarias. En el ciclo escolar 2020-2021 sienten que las fechas están más claras y persiste la esperanza del regreso a clases presenciales.

En el Estado de México, las/os docentes tuvieron que aprender los elementos básicos de la plataforma Google Meet, así como orientaron a los/as estudiantes para su uso y para la apertura de correos electrónicos. Las/os docentes estaban obligados a seguir las directrices establecidas a nivel estatal; sin embargo, gran parte de sus estudiantes no contaban con los recursos necesarios para trabajar en línea. Si bien ellas/os tuvieron claridad en las herramientas a utilizar, se sentían presionados/as para cumplir con dichas directrices, aunque no fueran adecuadas para sus estudiantes; en especial, subir las planeaciones, evidencias de trabajo y evaluaciones a la plataforma. 
Las/os docentes apreciaban que hubiera cursos de capacitación y eventos para abordar la educación a distancia; sin embargo, en su opinión, fueron excesivos, no todos de utilidad y se sumaban a sus pesadas actividades de docencia.

Las/os responsables de los planteles del Estado de México sentían que había una excesiva carga burocrática que ahora se dificultaba por realizarla en línea. Las plataformas no funcionaban adecuadamente y se les exigía el acopio de información para generar estadísticas. Además de los cursos de capacitación, se les exigía asistir a eventos oficiales del Gobierno del estado y de la SEMS, o incluso retuitear declaraciones de políticos, y para ello, estar disponibles en cualquier momento. Ellas/os, a su vez, se sintieron presionadas/os por los resultados que debían lograr, y en su opinión, no se tomaban en cuenta las condiciones de vulnerabilidad económica y académica de los/as estudiantes de TBC.

En el caso de Morelos, las/os propias/os responsables de los planteles y las/os docentes tuvieron que tomar todas las iniciativas y decisiones. Fueron ellas/os quienes elaboraron una estrategia para llevar a cabo la educación a distancia y quienes eligieron los medios más apropiados para las condiciones de la comunidad y de las/os estudiantes. Al no contar con recursos ni apoyos por parte del estado, acudieron a las plataformas de la SEMS, a cursos de capacitación que ofreció la Fundación Carlos Slim o aprendieron por su cuenta. La carga para las/os y responsables fue excesiva, pues fueron los encargados de los procesos de inscripción y evaluación, realizados por primera vez en línea. Si bien contaron con un margen de libertad mayor, se enfrentaron a la educación a distancia sin recursos ni apoyos.

\subsection{El programa Aprende en casa desde las comunidades rurales}

Una vez declarado el confinamiento sanitario y, por ende, el cierre de las escuelas, las/os docentes se dieron a la tarea de contactar a las/os estudiantes por cualquier medio: teléfono, Whatsapp, Facebook o a través de compañeros/as. La propuesta basada en los programas televisivos muy pronto se enfrentó con la realidad de las comunidades rurales. A pesar de que la cobertura televisiva es amplia en el país, no llegaba la señal para captar los canales en los que se transmitían los programas, o bien se requería una antena o televisión de paga. Ante este hecho, las/os docentes tuvieron que perfilar sus propias estrategias.

En el caso de Morelos, las estrategias narradas por las/os docentes fueron múltiples. La mayoría de ellas/os utilizaron Whatsapp o correo electrónico para enviar guías elaboradas por ellas/os mismas/os, tareas, materiales de apoyo y enlaces para videos. Por este mismo medio, las/os estudiantes regresaban la foto de los ejercicios resueltos. El correo electrónico fue utilizado en menor medida y las videoconferencias solo en los casos en que las/os estudiantes contaran con señal. Como se mencionó antes, en el Estado de México desde el inicio utilizaron la plataforma de Google Meet y las/os docentes buscaron otras opciones cuando las/os estudiantes no tenían acceso a esta.

Los problemas más frecuentes que enfrentaron tanto docentes como estudiantes con respecto a la educación a distancia de emergencia fueron el deficiente servicio de luz en gran parte de las localidades, la falta de conectividad a internet y la de señal telefónica. Otro problema fue que las/os estudiantes rara vez disponían de 
una computadora personal, cuando más, en casa había una que tenía que compartirse con otros miembros de la familia. Lo mismo sucedió con los teléfonos celulares, pues no todos/as las/os estudiantes contaban con un dispositivo y, por ello, también había que compartirlo. Adicionalmente, las familias no podían sufragar el gasto del teléfono, sobre todo cuando había varias/os hijas/os en edad escolar, y además los dispositivos no tenían capacidad para acceder al software y a las herramientas que requerían. ${ }^{5}$

Las condiciones de las/os docentes, en términos generales, fueron mejores que las de las/os estudiantes; sin embargo, los primeros tuvieron que sufragar los gastos de luz, internet y del saldo del celular que se consumían para actividades escolares. Se presentaron casos de docentes que no tenían computadora personal o en cuyas comunidades se presentaban fallas constantes del internet. En un principio, tenían dificultades para el manejo de las plataformas, pero poco a poco las fueron superando. Los problemas principales para ellos estaban relacionados con el exceso de carga de trabajo y los compromisos familiares derivados del trabajo doméstico y del cuidado de sus hijas/os.

Ante los obstáculos, las/os docentes buscaron en todo momento adaptarse a las condiciones de las/os estudiantes y lidiar con sus propias responsabilidades. En todos los casos se trataba de retroalimentar las tareas y aclarar las dudas. Al paso del tiempo, las/os docentes fueron cambiando sus estrategias después de valorar los resultados. Por su lado, las/os estudiantes desplegaron estrategias propias: buscaron conectarse al wifi en lugares públicos y enviar los trabajos por las noches, cuando había mejor señal. A pesar de los enormes esfuerzos, las/os jóvenes sentían que no aprendían y sus maestros/as se daban cuenta de que muchas veces ellas/os cumplían, pero no comprendían a cabalidad o se quedaban con dudas.

En cuanto al currículo, la SEP priorizó cumplir con los "aprendizajes esperados" establecidos para cada asignatura y grado; además puso énfasis en continuar con la secuencia y los contenidos de los libros de texto. En un principio, las/os docentes intentaron incorporar temas relacionados con la pandemia en contenidos de biología, ciencias de la salud y estadística, con la finalidad de generar el interés de las/os estudiantes. No obstante, ante el alargamiento de la pandemia, después se centraron solo en lo que consideraban más importante.

Las/os docentes señalaron que las áreas más difíciles de trabajar a distancia fueron matemáticas, química y física. El desarrollo de los proyectos comunitarios, que son uno de los pilares del modelo de TBC, tuvieron que cancelarse ante el confinamiento sanitario, ya que requerían trabajo directo con la comunidad. Solo se pudieron abordar los aprendizajes teóricos, mientras que las actividades llamadas paraescolares -normalmente deporte o baile- también tuvieron que suspenderse.

El programa Jóvenes en casa, que efectivamente abría un espectro de actividades recreativas y de apoyo socioemocional, tampoco tuvo el impacto esperado en las comunidades rurales. En ninguno de los TBC consultados fue utilizado por las/ os estudiantes por las mismas razones: falta de recursos para acceder a internet y escasez de tiempo.

\footnotetext{
${ }^{5}$ Los problemas descritos y las estrategias desplegadas por docentes y estudiantes coinciden con Mejoredu (2021) y con los resultados de la encuesta de Mejoredu (2020b) en lo que corresponde al nivel medio superior para los municipios con alta y muy alta marginación.
} 
Los libros de texto, por su carácter impreso, podrían haber sido un recurso muy importante para los TBC en la etapa de educación a distancia de emergencia y, sobre todo, de gran utilidad para las/os jóvenes que no tienen acceso a internet. Sin embargo, no fue así, ya que a partir de 2018 hubo un cambio en los planes y programas de estudio: se pasó de una organización por asignaturas a módulos, pero los libros de texto no se actualizaron. Para los/as docentes resultaba muy complicado identificar los contenidos, los ejercicios y las actividades que correspondieran a los del programa; por lo tanto, los utilizaron solo como un recurso complementario cuando había coincidencia.

La evaluación de los aprendizajes fue uno de los aspectos de mayor preocupación para las/os docentes. Ellas/os observaban a diario las dificultades de las/os jóvenes para adaptarse a la modalidad a distancia; la falta de condiciones de sus hogares para trabajar y para conectarse en línea, así como la poca motivación hacia los estudios. Por estas razones, les resultaba difícil establecer parámetros para calificar; intentaron ser flexibles y empáticos con la situación de las/os estudiantes, pero también se percataban de quiénes se esforzaban, quiénes eran indiferentes y quiénes nunca se comunicaron. Asimismo, procuraron seguir las recomendaciones emitidas por la SEP de tratar de no generar angustia entre las/os estudiantes, pero sí dejar clara la validez de la evaluación.

Uno de los parámetros que tuvieron que acatar las/os docentes a nivel federal fue no reprobar estudiantes en el ciclo escolar. Cuando se emitió dicho lineamiento se esperaba realizar un periodo de regularización para quienes presentaran mayores dificultades o que no se hubieran comunicado. Sin embargo, dicho plan no pudo llevarse a cabo por falta de condiciones sanitarias y se permitió continuar con los estudios a quienes así lo desearan. El criterio para asignar la calificación se basó en si se cumplió con la mayor parte del trabajo, se hizo de manera irregular o no hubo trabajo. Las/os docentes no llevaron a cabo la evaluación con base en la carpeta de evidencias propuesta en el programa Aprende en casa y que se integraría con los ejercicios y actividades recomendados por las emisiones televisivas.

El inicio del programa Aprende en casa I/ para el ciclo escolar 2020-2021, basado en la estrategia de ampliación de la cobertura televisiva, anunciaba claramente la continuidad de algo que había fracasado. Lo que se requería no eran más canales, sino otro tipo de apoyos. Había evidencias suficientes de las dificultades de acceso a internet en el medio rural, falta de dispositivos y de recursos para pagar la conectividad. No se escucharon las voces críticas que apuntaban el riesgo de establecer una estrategia única que resultaba insuficiente para sortear las profundas desigualdades sociales y económicas (Fernández y De la Rosa, 2020). Oliver (2020) advertía que los convenios con las televisoras, lejos de empoderar a las comunidades escolares reforzaban un modelo privatizador en el cual las/os docentes y las familias terminan pagando los insumos educativos. Se esperaba una estrategia gubernamental con inclusión digital, ampliación de internet, entrega de dispositivos tecnológicos -como se hizo en Argentina, Chile, Colombia, El Salvador, Jamaica, Perú, San Vicente y las Granadinas y Uruguay (Cepal-Unesco, 2020)- y de tarjetas de prepago para internet y datos del celular tanto a docentes como a estudiantes. 
El ciclo escolar dio inicio con los mismos libros de texto, pero a pesar de que se distribuyeron a tiempo, no se actualizaron conforme a los nuevos planes y programas de estudio y, por tanto, se presentaron los mismos problemas que en la etapa previa. Una de las novedades del programa Aprende en casa Il fue la oferta de un segmento de la programación diseñada especialmente para TBC. Este hecho, que de alguna manera significaba un avance, no tuvo el impacto esperado. Ello se debió, por una parte, a que los problemas para el acceso a la señal televisiva fueron los mismos que en el ciclo escolar anterior y, aunque se podían visualizar los programas desde la plataforma de la SEMS, difícilmente las/os jóvenes podían acceder a ésta. Por otra parte, los contenidos de las transmisiones no coincidían con los planes y programas de estudio y además no se encontraban diferenciados por grado. En las comunidades en las que había señal de televisión, a las/os docentes se les dificultaba utilizar los programas televisivos, ya que no se podía hacer una planeación, pues el contenido no se daba a conocer previamente. Adicionalmente, los horarios de las emisiones no se adaptaban a los/as estudiantes que debían trabajar, puesto que se ofrecían un primer turno de 12:00 a 14:00 h y una repetición de 22:00 a 24:00 h, horario que tanto docentes como estudiantes consideraban ser excesivamente tarde. Las/los estudiantes tampoco siguieron el programa Jóvenes en casa por falta de interés y de recursos de acceso a la plataforma.

En cuanto al desarrollo de los proyectos comunitarios, en ambas entidades federativas hubo docentes que encontraron formas creativas de adaptarlos; por ejemplo, mediante actividades realizadas en casa o elaboración de materiales digitales para la promoción de la salud. El espacio de las actividades paraescolares se utilizó para tutorías personales o grupales de apoyo emocional y académico, o bien para conferencias y talleres relacionados con temas de interés, como la violencia de género o la salud.

Siguiendo la misma estrategia gubernamental centrada en la televisión, quienes generaron los cambios fueron los propios docentes y estudiantes. Durante el periodo vacacional, las/os docentes se prepararon, tomaron cursos de capacitación y regresaron a clases con mejores habilidades digitales y algunos de ellos con nuevo equipo telefónico o de cómputo. Tomaron como base la experiencia del ciclo anterior y ajustaron la organización. Entre los cambios más notables que reportaron las/os docentes de ambas entidades federativas se pueden mencionar los siguientes: 1) utilizaron una plataforma única por plantel; 2) aprovecharon las distintas aplicaciones para alojar el material de apoyo y las tareas, con el fin de que las/os estudiantes pudieran utilizarlos a cualquier hora o cuando tuvieran internet; 3) dosificaron las videoconferencias en frecuencia y duración, y recurrieron a estas solo para los aspectos que se consideraban necesarios; 5) seleccionaron con mayor cuidado los videos de apoyo para que fueran más claros y cortos; 4) utilizaron Whatsapp solo para información operativa con las/ os estudiantes y la familia; 5) asignaron a cada área del conocimiento un día y establecieron claramente la fecha de entrega de trabajos; 6) equilibraron las tareas que cada docente asignaba para no exceder la carga de trabajo de las/os estudiantes; 7 ) establecieron horarios de atención a estudiantes; 8) definieron criterios de evaluación más claros y flexibles; 9 ) crearon estrategias diferenciadas por grado y para las/os 
jóvenes con mayores dificultades -en algunos casos incluían asesorías presenciales-, y 10) generaron apoyos externos de asesoría en sistemas informáticos, conferencias y cursos de formación técnica para las/os estudiantes.

Las/os estudiantes, por su parte, también emprendieron cambios: las familias hicieron esfuerzos para contratar internet que no implicaran el pago de recargas de celular; trabajaron y utilizaron su beca para comprar celulares; se organizaron en grupos de cuatro a cinco estudiantes para reunirse en una casa con internet, o se turnaban para ocupar el saldo de uno/a y no de cinco jóvenes. Las medidas tomadas por las/os docentes también fueron de utilidad para las/os jóvenes a fin de organizar su tiempo y minimizar los costos.

\section{Conclusiones}

A lo largo de este artículo se ha mostrado que la política educativa desplegada durante la pandemia con el programa Aprende en casa fue más mediática que de impacto en la educación a distancia de emergencia en las escuelas. Los efectos más visibles se observaron en el plano normativo más que en la creación de las condiciones, los insumos, la infraestructura y el acompañamiento didáctico-pedagógico a docentes y estudiantes. A pesar de las limitaciones de este programa y de los constantes cambios y de la incertidumbre, fue útil para las/os responsables de los planteles, docentes, estudiantes y familias contar con un referente normativo en torno a la validez de los estudios, los calendarios de los ciclos escolares y los parámetros a seguir para el regreso a las clases presenciales. Sin embargo, las/os docentes refieren un mayor interés de la SEP por cumplir con los contenidos y con acreditar el ciclo escolar que por los aprendizajes de las/os estudiantes.

La estrategia del programa Aprende en casa partió de una idea homogénea de la escuela y no de las condiciones concretas del medio rural; se centró en la programación televisiva porque partió de la premisa de la extensa cobertura nacional sin tomar en cuenta el acceso a la señal de televisión. Tampoco se tomó en cuenta que, para establecer la comunicación entre docentes y estudiantes se requería de dispositivos, servicios de luz e internet adecuados. En este sentido, el Gobierno federal perdió la oportunidad de cambiar o ajustar el programa a partir de las evidencias y de las experiencias de lo que había sucedido en los TBC. Se esperaba un nuevo programa que tomara en cuenta las particularidades del nivel medio superior y de las zonas rurales, que apoyara con recursos a docentes y estudiantes y que se trabajara en la ampliación de la conexión a internet.

El programa Aprende en casa tenía problemas básicos de diseño y de operación que dificultaban aún más su uso. Tales fueron los casos de la falta de difusión de la programación previa, de la diferenciación por grados, horarios inadecuados y escasa correspondencia con los contenidos de los planes y programas de estudio. Lo mismo sucedió con los libros de texto, que se convirtieron en un simple complemento al no estar actualizados. El programa Jóvenes en casa, que se dirigía justamente a la población de los bachilleratos, tampoco pudo utilizarse por las inadecuadas condiciones de acceso a la plataforma de la SEMS. De esta manera, las/os responsables de plantel y las/os docentes se quedaron solos y sin herramientas para trabajar. 
A nivel meso, la política estatal, que pudo haber llenado los vacíos del programa y orientarse a cubrir las necesidades particulares de los bachilleratos rurales, solo se cumplió parcialmente. En el Estado de México se siguió una política clara que marcó directrices de operación y seguimiento de la educación a distancia de emergencia; aunque con frecuencia inadecuadas para los TBC. Tanto las/os responsables de plantel como las/os docentes tuvieron parámetros para operar, pero también efectos contraproducentes, como el control, la presión y la burocratización de las tareas. En el caso de Morelos, toda la responsabilidad recayó sobre directivos/as y docentes.

Ante el vacío en el que quedaron los TBC, el peso de la educación a distancia de emergencia lo asumieron, en gran parte, las/os responsables y docentes con sus propios recursos, creatividad y compromiso. Fueron ellos quienes propiciaron e hicieron posibles los cambios y los ajustes en las distintas etapas. La responsabilidad también recayó en las familias más vulnerables, que asumieron el costo de la conexión a internet y de los dispositivos de sus hijos. Un lugar merecen igualmente las/os estudiantes al adaptarse a una modalidad de enseñanza que no les gustaba y para la que no contaban con los recursos adecuados.

\section{Referencias}

Comisión Económica para América Latina-Organización de las Naciones Unidas para la Educación, la Ciencia y la Cultura. (2020). La educación en tiempos de COVID-19. Informe COVID-19. Cepal-Unesco. Recuperado de https://bit.ly/3wzi9lB

Comisión Nacional para la Mejora Continua (2020a). Indicadores nacionales de la mejora continua de la educación en México 2020. Cifras del ciclo escolar 2018-2019. Gobierno de MéxicoMejoredu. Recuperado de https://bit.ly/3fKu7si

Comisión Nacional para la Mejora Continua de la Educación. (2020b). Comunidades escolares al inicio del confinamiento por SARS-CoV-2. Voces y perspectivas de los actores. Mejoredu. Recuperado de https://bit.ly/3i7r3t9

Comisión Nacional para la Mejora Continua de la Educación. (2021). Experiencias de las comunidades educativas durante la contingencia por Covid-19. Educación Media Superior. Mejoredu. Recuperado de https://bit.ly/2REaf23

Estrada, M. y Alejo, S. J. (2019). De la cobertura a la búsqueda de equidad. Universidad de GuanajuatoColofón.

Fernández, M. A y De la Rosa, R. (28 de agosto de 2020). Ante la precaria inclusión digital, ¿aprender en casa? Nexos. Distancia por tiempos. Recuperado de https://bit.ly/3yDp8IG

Flick, U. (2004). Introducción a la investigación cualitativa. Morata.

Flores-Crespo, P. (2011). Análisis de política educativa: un nuevo impulso. Presentación temática. Revista Mexicana de Investigación Educativa. 16 (8), 687-698. Recuperado de https:// bit.ly/3wziktP

Gibbs, G. (2012). El análisis de datos cualitativos en investigación cualitativa. Morata.

Gobierno Federal (2021). Coordinación Nacional de Becas para el bienestar Benito Juárez. Recuperado de https://bit.ly/3fNTmtK

Guzmán, C. (2018). Avances y dificultades en la implementación del Marco Curricular Común. Telebachillerato estatal, educación media superior a distancia y telebachillerato comunitario. Instituto Nacional para la Evaluación de la Educación. Recuperado de https://bit.ly/3ylObWb

Huberman, M. y Miles, M. (2000). Métodos para el manejo y el análisis de los datos. En C. Denman y J. Haro (comps.). Por los rincones. Antología de métodos cualitativos en la investigación social (pp. 253-300). Colegio de Sonora. 
Infobae (3 de agosto de 2021). Somos pioneros en este sistema: López Obrador sobre el convenio con Emilio Azcárraga y Salinas Pliego apara el regreso a clases. Infobae. Recuperado de https://bit.ly/3vnp1J9

Instituto Nacional de Estadística y Geografía. (2019). Encuesta Nacional sobre Disponibilidad y Uso de Tecnologías de la Información en los Hogares (ENDUITH). INEGI. Recuperado de https://bit.ly/3filc1Y

Instituto Nacional para la Evaluación de la Educación. (2019). La educación obligatoria en México. Informe 2019. Instituto Nacional para la Evaluación de la Educación. Recuperado de https://bit.ly/2QRAbXo

Instituto Nacional para la Evaluación de la Educación, Instituto Internacional de Planeamiento de la Educación y Organización de las Naciones Unidas para la Educación, la Ciencia y la Cultura. (2018). La política educativa en México desde una perspectiva regional. Instituto Nacional para la Evaluación de la Educación. Recuperado de https://bit.ly/3hVNa5w.

Oliver, L. (26 de agosto de 2020). Aprende en Casa II: cuando la construcción de capacidades locales no importa. Nexos. Distancia por tiempos. Recuperado de https://bit.ly/34kle2e

Organización de las Naciones Unidas. (2020). Informe de políticas: La educación durante la COVID-19 y después de ella. ONU. Recuperado de https://bit.ly/3oRJW4j

Pedró, F. (2020). COVID-19 y educación superior en América Latina y el Caribe: efectos, impactos y recomendaciones políticas. Análisis Carolina. 36/2020. https://doi.org/10.33960/ AC_36.2020

Secretaría de Educación Pública. (30 de marzo 2020a). Boletín 75. Gobierno de México. Recuperado de https://bit.ly/3oRJW4j

Secretaría de Educación Pública. (18 de abril de 2020b). Boletín 98. Gobierno de México. Recuperado de https://bit.ly/3hTEZql

Secretaría de Educación Pública. (25 de agosto de 2020c). Boletín 236. Gobierno de México. Recuperado de https://bit.ly/3bVjQIC

Secretaría de Educación Pública. (29 de agosto 2020d). Boletín 240. Gobierno de México. Recuperado de https://bit.ly/3hSpMG7

Secretaría de Educación Pública. (9 de septiembre de 2020e). Boletín 247. Gobierno de México. Recuperado de https://bit.ly/3yDJ5Jc

Secretaría de Educación Pública. (23 de septiembre de 2020f). Boletín 256. Gobierno de México. Recuperado de https://bit.ly/3fkyFGA

Secretaría de Educación Pública. (25 de septiembre de 2020g). Boletín 258. Gobierno de México. Recuperado de https://bit.ly/3vqjJwh

Secretaría de Educación Pública. (23 de octubre de 2020h). Boletín 275. Gobierno de México. Recuperado de https://bit.ly/3vo5iJc

Secretaría de Educación Pública. (27 de diciembre de 2020i). Boletín 332. Gobierno de México. Recuperado de https://bit.ly/34kfgiM

Secretaría de Educación Pública, Subsecretaría de Educación Media Superior y Dirección General de Bachillerato. (2020). Telebachillerato Comunitario. México: SEP-SEMS-DGB. https:// bit.ly/3uoZzRU

Senado de la República (14 de octubre de 2020). Boletín 264. El sistema educativo a la altura del desafío de la pandemia, informa Esteban Moctezuma en el senado. Gobierno de México. Recuperado de https://bit.ly/34jAOau

Stake, R. (2010). Investigación con estudio de casos. Madrid: Morata.

Vasilachis de Gialdino, I. (2006). La investigación cualitativa. En: I. Vasilachis de Gialdino (coord.). Estrategias de investigación cualitativa (pp. 23-106). Gedisa.

Weiss, E. (2017). Estudio exploratorio del Modelo de Telebachillerato Comunitario y su operación en los estados (Cuaderno de Investigación 47). Instituto Nacional para la Evaluación de la Educación. Recuperado de https://bit.ly/3hUtcYT

World Economic Forum (2016). Informe 2015-2016. World Economic Forum. Recuperado de https:// bit.ly/3fmx9Uv 